\title{
Continuous dynamic assimilation of the inner region data in hydrodynamics modelling: optimization approach
}

\author{
F. I. Pisnitchenko ${ }^{1}$, I. A. Pisnichenko ${ }^{2}$, J. M. Martínez ${ }^{1}$, and S. A. Santos ${ }^{1}$ \\ ${ }^{1}$ State University of Campinas, Campinas, SP, Brazil \\ ${ }^{2}$ Center for Weather Forecast and Climate Studies/National Inst. for Space Research - INPE, Cachoeira Paulista, SP, Brazil
}

Received: 22 February 2008 - Revised: 30 May 2008 - Accepted: 9 September 2008 - Published: 3 November 2008

\begin{abstract}
In meteorological and oceanological studies the classical approach for finding the numerical solution of the regional model consists in formulating and solving a CauchyDirichlet problem. The boundary conditions are obtained by linear interpolation of coarse-grid data provided by a global model. Errors in boundary conditions due to interpolation may cause large deviations from the correct regional solution. The methods developed to reduce these errors deal with continuous dynamic assimilation of known global data available inside the regional domain. One of the approaches of this assimilation procedure performs a nudging of large-scale components of regional model solution to large-scale global data components by introducing relaxation forcing terms into the regional model equations. As a result, the obtained solution is not a valid numerical solution to the original regional model. Another approach is the use a four-dimensional variational data assimilation procedure which is free from the above-mentioned shortcoming. In this work we formulate the joint problem of finding the regional model solution and data assimilation as a PDE-constrained optimization problem. Three simple model examples (ODE Burgers equation, Rossby-Oboukhov equation, Korteweg-de Vries equation) are considered in this paper. Numerical experiments indicate that the optimization approach can significantly improve the precision of the regional solution.
\end{abstract}

\section{Introduction}

Studying and modelling different physical processes frequently require to solve Cauchy-Dirichlet problems. In geophysical investigations, for solving the Cauchy-Dirichlet

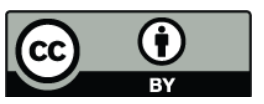

Correspondence to: F. I. Pisnitchenko (feodor@ime.unicamp.br) problem, numerical methods are usually employed. The discrete form of equations and initial and boundary values at the points of the grid are traditionally used in these methods. The problem is generally solved by means of a proper numerical scheme. However, both initial and boundary values, which are obtained from measurements or other model outputs, contain errors, which can reach $30 \%$ from the true values.

For some problems, the solution can be very sensitive to these errors. On the other hand, some values of the sought solution at some points of the inner region, along with initial and boundary data, are often available. Of course, these additional data also have errors. The question arises: is it possible to improve the accuracy of the model solution of the Cauchy-Dirichlet problem by using these additional data? For some well-known equations of mathematical physics and dynamic meteorology we will show here that the use of additional information on the solution values within the integration region can noticeably improve the accuracy of the solution.

Improving solution accuracy of the Cauchy-Dirichlet problem is extremely important in meteorology because this is the problem that arises in regional weather forecast. A distinction between two types of atmospheric forecast models needs to be done. Global models deal with forecast for the whole earth, and regional models produce a forecast for a limited area. One of the basic differences between these model types is the grid resolution. Global models use a low resolution space grid and regional models operate on a more dense mesh. The reason for the existence of these two model types is, basically, computational. Even nowadays it is not possible in acceptable time period to integrate global models with the detailed physics and with the space resolution of regional models. It is worth noticing that global and regional models include different physical processes. Global models describe large-scale slow-time varying processes, with time

Published by Copernicus Publications on behalf of the European Geosciences Union and the American Geophysical Union. 
period of more than $3 \mathrm{~h}$ and space scale larger than $60 \mathrm{~km}$. Regional models can simulate the evolution of mesometeorological fast processes (small cyclones, storms, tornados), with time period of less than $3 \mathrm{~h}$ and spatial extent between 2 and $60 \mathrm{~km}$.

These models are described mathematically by systems of nonlinear partial differential equations. To solve the system corresponding to a global model we need only the initial condition and bottom and upper boundary conditions, since the domain is the whole sphere. To get the solution of a system corresponding to a regional model we also need lateral boundary conditions. The lateral boundary conditions for a regional model are obtained from the global model using interpolation. These conditions do not provide information on the structures of the scales smaller than the size of the mesh of the global model. In fact, global models do not distinguish a local meteorological phenomenon of characteristic length scale smaller than $30 \mathrm{~km}$, because the space step is greater than this value. On the other hand, the parts of space and time spectrums of a regional model solution which correspond to large-scale structures and long-period processes are in worse accordance with observations than those of a global model. This is related to the fact that a regional model does not have the information about the phenomena that occur outside its domain.

For example, the weakly regular, low frequency oscillations of the sea surface temperature of tropical Pacific in central part and near the Peru coast, known as El Niño and La Niña events, have a large influence on the climate of the tropical and subtropical regions. The circulation regimes in these regions noticeably differ during these events. Regional models, driven, for example, by the Reanalysis data, do not reproduce with confidence this distinction between the El Niño and La Niña regimes (Seth et al., 2007; Gershunov et al., 2000). The reason of this inconsistency is, probably, poor space and time data resolution on the regional model boundaries. Mathematically, this means that the solution of this boundary value problem is strongly sensitive to errors on the boundaries (Denis, Laprise and Caya, 2003; Diaconescu, Laprise and Sushama, 2007).

The spectral nudging technique is one of methods proposed to use additional data from the inner domain in order to reduce boundary error influence and to improve the solution of an initial-boundary value problem (Waldron et al., 1996; Storch et al., 2000; Kanamaru and Kanamitsu, 2005). This method supposes incorporation of the largest internal modes of some meteorological variables from observations or from a driving model into the regional model solution. However, the use of the spectral nudging technique requires inserting additional forcing terms into the evolution equations of a regional model. Hence, the original model is corrupted and its new solution may not be close to the correct regional solution.
Another method which is aims to avoid strong sensitivity of regional model solution with respect to the boundary condition errors is based on four-dimensional variational (4DVar) data assimilation procedures (Le Dimet and Talagrand, 1986; Rabier, 2005; Lorenc and Payne, 2007). In the papers of Leredde et al. (1998), Zou and Kuo (1996), Lu and Browning (2000) and Griffin and Thomson (1996) optimization approach is applyed to restore boundary conditions using data within spatial model domain. 4D-Var performs an adjustment of the model trajectory with all available data (observations or global model data) taken explicitly at the precise time and thus the obtained model solution is maximally possibly consistent with available observations.

In this work we apply the optimization theory to the 4DVar data assimilation procedure. Firstly, in Sect. 2 we give the formulation of the Cauchy-Dirichlet problem for a regional model and the general formulation of an optimization problem, and we show how the latter (with the accessibility of some additional conditions) can be applied for finding the solution of the former. In Sect. 3, for some simple equations, we show how the optimization approach can be used to obtain a solution of the initial boundary value problem. The equations considered here include the ordinary differential equation of Burgers, the one-dimensional linearized RossbyOboukhov partial differential equation, and the partial differential equation of Korteweg - de Vries. We also compared the sensitivity of the solutions, obtained by the traditional and the new approach with respect to the errors in the boundary conditions. In Sect. 4 we discuss the obtained results. Appendix A provides more detailed description of the optimization procedure.

\section{Numerical solution of regional problems}

\subsection{Classical approach}

We formulate here the Cauchy-Dirichlet problem as it is stated for regional weather forecast. To find a unique solution for the regional model equations we need the lateral boundary condition for the entire time interval on which the solution is required. Usually, these data are obtained from the solution of an outer (global) model. We do not know the exact solution of the outer model (otherwise, our problem would have been already solved), but we can find its approximate solution in the discrete form:

$$
\begin{aligned}
& \Delta_{t}\{\Psi\}=F_{d}, \\
& \Psi(x, 0)=Y_{\text {global }}(x), \\
& \left.\Psi(x, t)\right|_{x \in B}=\Psi_{b}(x, t) .
\end{aligned}
$$

Here $\Delta_{t}$ is the evolution operator of the discrete model, $\Psi$ is the vector of the prognostic functions, $F_{d}$ are discrete external forces, $Y_{\text {global }}$ is the initial condition, $B$ are upper, 


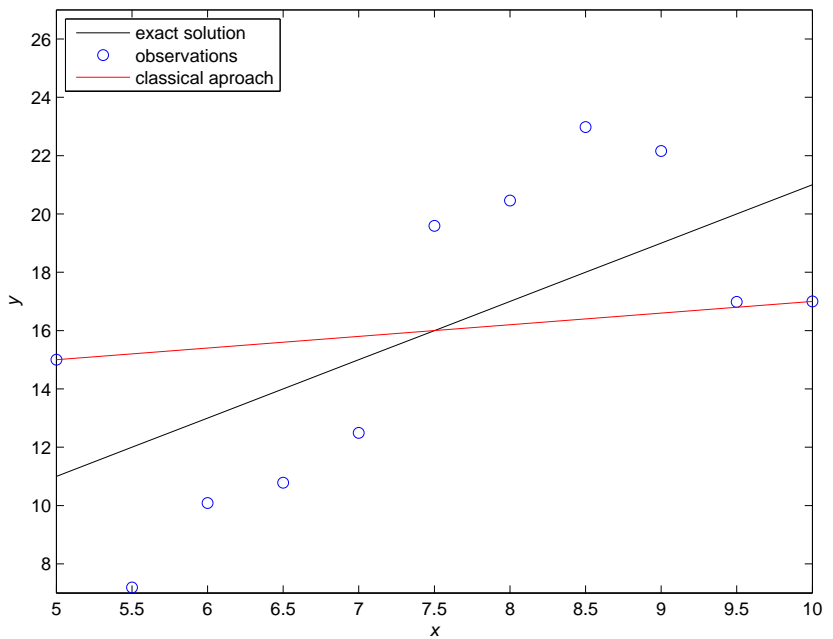

Fig. 1. Solution of $y^{\prime \prime}=0$ using classical approach.

lower (and maybe lateral) boundaries of the global model, and $\Psi_{b}$ is the boundary condition. Let $\Psi_{\text {sol }}$ be the solution of (1). It is supposed that this solution is sufficiently close to the solution of hypothetical ideal model which exactly describes the real atmospheric processes.

The regional model is located in a closed area with boundary $S$ and its discrete representation can be written in the same manner as for the global model:

$$
\begin{aligned}
& \delta_{t}\{G\}=F_{r d}, \\
& G(x, 0)=Y_{\text {local }}(x), \\
& \left.G(x, t)\right|_{x \in S}=G_{s}(x, t),
\end{aligned}
$$

where $\delta_{t}$ is the evolution operator of the discrete regional model, $G$ is the vector of the prognostic functions and $F_{r d}$ are discrete external forces, $Y_{\text {local }}$ is the initial condition, and $G_{s}$ is the boundary condition.

The traditional approach to seek the solution of (2) consists in the interpolation of required data from $\Psi_{\text {sol }}$ on the regional grid for forming the boundary and initial conditions, and then applying any numerical method to solve the model equations. However, as we have mentioned above, the boundary conditions contain errors which can strongly corrupt the solution.

The use of the information obtained from the solution of the global model equations $\Psi_{\text {sol }}$ on the values inside the regional model domain for all available time moments can help to overcome this difficulty.

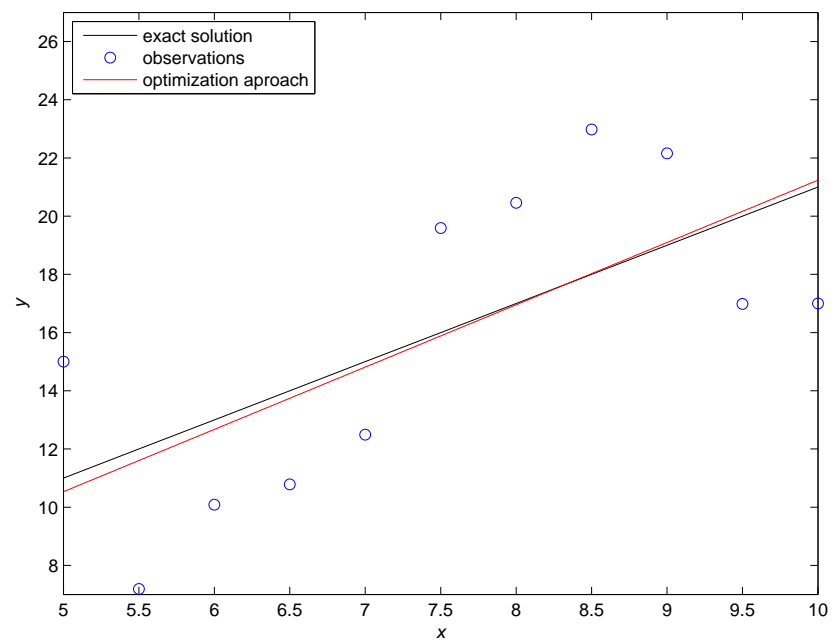

Fig. 2. Solution of $y^{\prime \prime}=0$ using optimization approach.

\subsection{Optimization approach}

As mentioned in the previous section, in the classical approach we obtain the boundary conditions for the regional model by means of interpolation of the coarse grid solution provided by the global model. Essentially, interior points also provided by the global model are discarded. The optimization approach consists of taking not only close-to-the boundary points on the global grid, but also global solution values at interior points. A trivial example will clarify this procedure and show its plausibility. Assume that $\left(x_{1}, y_{1}\right), \ldots,\left(x_{m}, y_{m}\right)$ are "global model" observations and that we want to fit the "local model" defined by $y^{\prime \prime}=0$. The "classical approach" would consist in taking, as solution, the line that joins $\left(x_{1}, y_{1}\right)$ with $\left(x_{m}, y_{m}\right)$. The optimization approach consists in finding the regression line that considers all the points. If the data were free of errors, both results would be identical, but, in the presence of errors, the difference may be dramatic. In Figs. 1 and 2 we show an example where the data $\left(x_{i}, y_{i}+e_{i}\right), i=1, \ldots, m$, corresponding to the exact solution $y=2 x+1$, were randomly perturbed, where $e_{i}$ represents a $\pm 30 \%$ of $y_{i}$. It is easy to see that only the optimization approach provides a reasonably accurate solution. We claim that the same situation occurs in more complicate mathematical environments.

The required solution of the regional model must satisfy the regional-model differential equations and the observed initial conditions. Therefore, the regional model solution must satisfy the constraints

$$
\begin{aligned}
& \delta_{t}\{G\}-F_{r d}=0 \\
& G(x, 0)-Y_{\text {local }}(x)=0 .
\end{aligned}
$$


Among the set of functions that satisfy these constraints we seek the one that minimizes the distance to the observed points $\Psi_{\text {sol }}$ produced by the global model. Therefore, we have the optimization problem:

Minimize $d\left(G, \Psi_{\text {sol }}\right)$

$$
\begin{aligned}
& \text { subject to (s.t.) } \delta_{t}\{G\}-F_{r d}=0, \\
& \qquad G(x, 0)-Y_{\text {local }}(x)=0 .
\end{aligned}
$$

Here $d(:,:)$ is the objective function, which represents the distance between $G$ and $\Psi_{\text {sol }}$. In other words, we want to minimize the distance between the regional and the global model solutions under constraints that say that the regional model equations and the initial condition are satisfied. Note here that, when we talk about the solution $G$, we bear in mind the vector $G=G(x, t)$ in a grid space of $(x, t)$ - coordinates, where $t$ corresponds to discrete time points of the regional model integration and $x$ to discrete mesh points in the regional model area.

For a long integration time, the dimension of the minimization problem can become too large, making it imposible to be solved. Then, instead of solving it as a unique problem, we split it in several minimization problems as follows. The time is divided in periods and, for each period, the related minimization problem has an appropriate dimension. The model is solved by means of the minimization problem in each period. For the first problem, the initial condition is taken from the "observations". For the second problem, the initial condition is the solution of the previous problem (first problem) in the lastest time level. And so on the minimization problems are solved until the last period.

The use of optimization algorithms for analysis and assimilation in meteorology has been considered by many authors. Modern optimization approach methods were discussed in the survey of Rabier (2005). One of the first and fundamental papers which placed the basis of application of optimization theory to meteorology and presented some algorithms for the realization of this method is the paper of Le Dimet and Talagrand (1986). This paper also includes detailed references on the previous works. The general idea of optimization approach is, always, to find the solution of a given model which is closest to a set of "observations". In our case, the "observations" must be interpreted as the solution of the global model. In (Le Dimet and Talagrand, 1986) the authors discuss, among other methods, the use of the Augmented Lagrangian methodology for solving the associated minimization problem. By means of Augmented Lagrangian methods the original problem is solved as a sequence of unconstrained optimization problems where the objective function includes information on the constraints. This approach is very adequate in general optimization for large scale problems in which the Jacobian information is badly structured, so that sparse matrix techniques for solving linear systems are hard to employ. The main disadvantage of the Augmented Lagrangian method is that the final convergence speed uses to be poor, although acceleration techniques have been recently introduced (?Birgin and Martínez, 2008). However, acceleration techniques are based on the direct consideration of the optimality (Euler-Lagrange) conditions of the system, and may be used in the cases in which sparsity can be handled. In many optimization problems the EulerLagrange nonlinear system is suitably structured and can be solved by means of Newtonian or quase-Newton techniques (Martínez, 2000). As a matter of fact, our first attack to the problems reported in this paper was by means of the Augmented Lagrangian, using the modern software Algencan (Andreani et al., 2007, 2008) In spite of being successful in obtaining solutions, we found that computer time was unacceptable and, so, we switched to the Newtonian technique.

In the paper of Le Dimet and Talagrand (1986) a different optimization approach is suggested that, in terms of our problem, may be formulated as "Find the initial and boundary conditions that make the solution of the PDE model to be closest to the observations". In this way, the objective function of the problem has only the initial and boundary conditions as independent variables, being therefore the dimension of the domain much smaller. However, the objective function computation involves the solution of the PDE system in this case and the derivatives with respect to the independent variables are generally necessary for its minimization. Fortunately, the technique based on the Adjoint System (Errico, 1997; Fisher et al., 2006) (which involves solving the linearized form of the model after computing the state variables) may be used to compute the gradient. This technique is very well known in Control problems and is generally effective. Moreover, it has inspired the so called Reverse Mode for Automatic differentiation of general functions currently used in Automatic Differentiation (AD) packages (Griewank, 1989; Birgin and Evtushenko, 1998). In their survey Le Dimet and Talagrand mention on the possible inaccuracy introduced by the disagreement between the continuous and the discretized form of the model, and that this inaccuracy can be removed using the Adjoint technique directly on the discretized model, as AD packages do. In our context, the disadvantage of this technique relies on the necessity of completely solving the model, for given boundary and initial conditions, which requires a stable PDE technique. Stability of the PDE techniques, on the other hand, is linked to the size of the time step, which needs to be, many times, unacceptably small. On the other hand, as we will see, small time steps are not required when one uses the Euler-Lagrange system, independently of the numerical scheme used to solve the PDE. 


\section{Examples of application of the optimization method to the problems of small dimension}

\subsection{Burgers' equation}

We will exhibit an application of the optimization method to problems of small dimension. As a first example we consider the supersensitive boundary value problem for the Burgers' equation (Bohé, 1996), used for describing wave processes in acoustics and hydrodynamics:

$$
\begin{aligned}
\epsilon x^{\prime \prime} & =-x x^{\prime}, \\
x(0) & =-1, x(T)=1,
\end{aligned}
$$

where $x$ is velocity and $\epsilon$ is the viscosity coefficient.

To get the analytical solution of this problem it is enough to integrate the left and right sides of the Eq. (5) over $t$ :

$$
\epsilon x^{\prime}=-\frac{x^{2}+c}{2}
$$

Then, after rewriting the foregoing formula as

$$
\epsilon \frac{d x}{x^{2}+c}=-\frac{d t}{2}
$$

and integrating the left side over $x$ and the right one over $t$, we can write the Burgers' equation solution as

$x(t)=\left\{\begin{array}{l}\frac{\sqrt{-c}\left(1+\exp \frac{\left(t+C_{2}\right) \sqrt{-c}}{\epsilon}\right)}{1-\exp \frac{\left(t+C_{2}\right) \sqrt{-c}}{\epsilon}}, \text { for } c \leq 0, \\ \sqrt{c} \tan \left(\frac{\left(t+C_{2}\right) \sqrt{c}}{2 \epsilon}\right), \text { for } c>0 .\end{array}\right.$

The graph of the analytical solution for Eq. (5) with the boundary condition $x(0)=-1, x(1)=1$ and $\epsilon=0.05$ is presented in Fig. 3 (black line).

Let us choose several points from the analytical solution and slightly perturb them (till 5\% from its real value). This procedure models the boundary and inner domain data containing errors. Using these perturbed data for the boundary points $x(0), x(1)$ we solve the boundary problem (5) applying the shooting method. For the step $\Delta t=0.01$ the numerical solution is presented in Fig. 3 by red line.

As one can see, small perturbations of the boundary condition result in large errors in the solution. Now, let us solve the problem using optimization formulation (4) with additional information about the perturbed solution on the points inside the domain, considering the same discretization as when solving (5) by the traditional shooting method, with the same $\Delta t=0.01$.

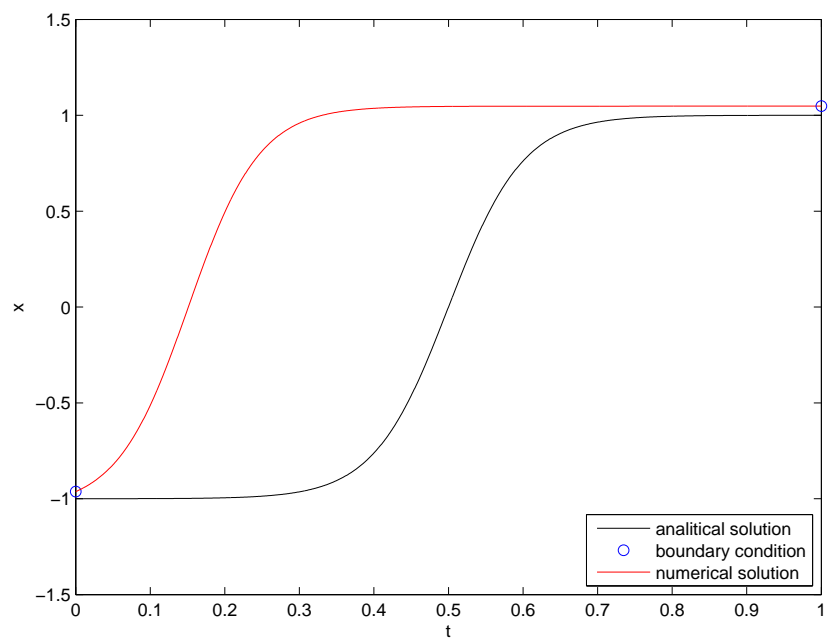

Fig. 3. Analitical solution (black line), using $x(0)=-1, x(1)=1$ as boundary condition, and numerical solution (red line), using $5 \%$ perturbed boundary condition (circles), of Burgers' equation.

Figure 4 shows the solutions of the optimization problem for the three cases when we used 3,4 and 5 points of the perturbed solution, respectively. One can see that all these solutions correspond better to the analytical one, than the solution obtained by the traditional approach. In the case of using the three additional inner points, the optimization solution and the analytical solution almost coincide. This example shows that there are dynamical systems in which small perturbations $(\leq 5 \%)$ in the boundary conditions can lead to great errors in the final solution. However, if some additional information exists, that information can be used to improve significantly the solution, applying optimization.

\subsection{Rossby-Oboukhov one-dimensional equation}

The two-dimensonal non-linear Rossby-Oboukhov equation describes the evolution of potential vorticity in barotropic atmosphere (Oboukhov, 1949). In this example we consider the one-dimensional linear equation obtained during the linearization procedure of the Rossby-Oboukhov equation.

$$
\frac{\partial}{\partial t}\left(\frac{\partial^{2}}{\partial x^{2}}-\frac{1}{l_{0}^{2}}\right) \psi+\beta \frac{\partial \psi}{\partial x}+U \frac{\partial^{3} \psi}{\partial x^{3}}=0 .
$$

Here $\psi$ is the stream function, $f_{0}=10^{-4} \mathrm{~s}^{-1}$ is mean value of Coriolis parameter, $\beta=d f / d y=1.6 \cdot 10^{-11} \mathrm{~s}^{-1} \mathrm{~m}^{-1}$ is mean value of meridional gradient of Coriolis parameter, $l_{0}=c_{0} / f_{0}=3 \cdot 10^{6} \mathrm{~m}$ is the Oboukhov scale, $c_{0}$ is the sound velocity, and $U$ is the zonal wind, which varies between 0 and $30 \mathrm{~m} / \mathrm{s}$. 

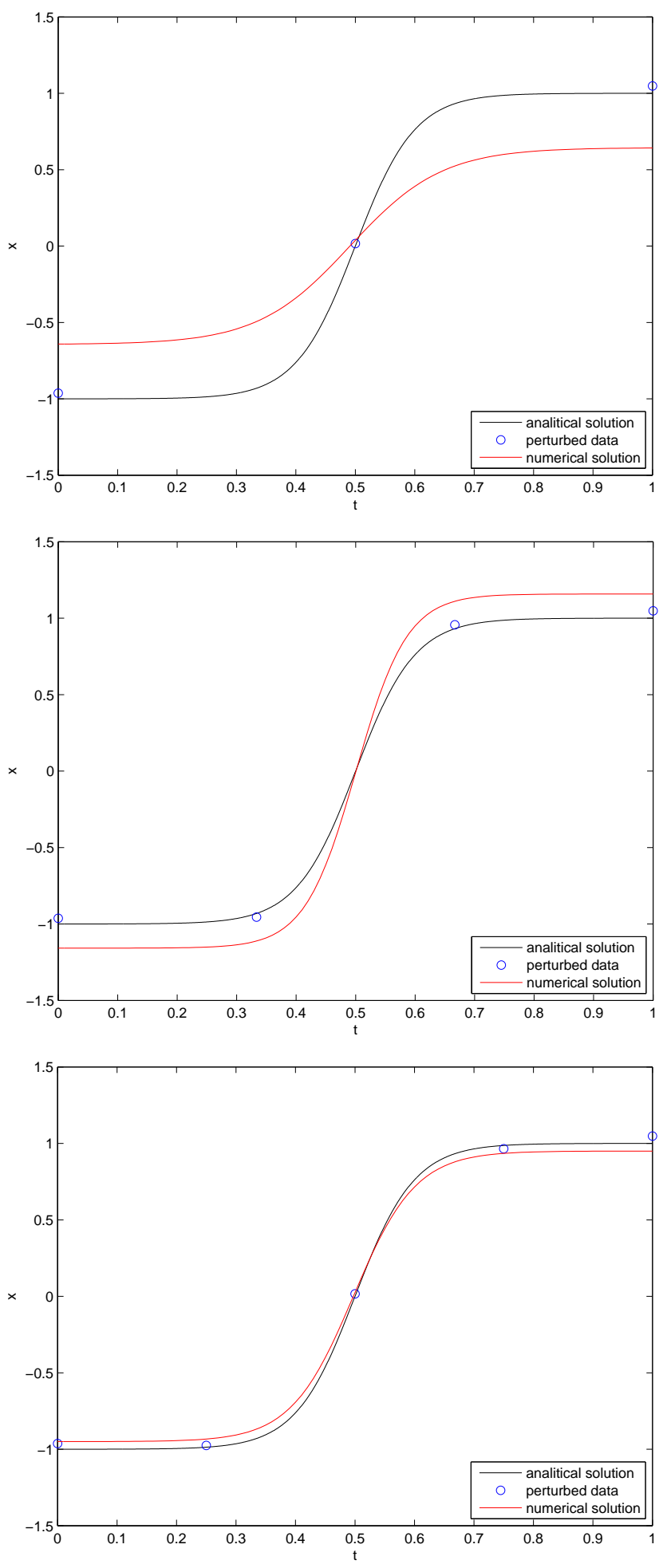

Fig. 4. Numerical solution of Burgers' equation using optimization formulation (red line) for three cases: 3 data points (upper panel); 4 data points (middle); and 5 data points (lower panel). Black line corresponds to analytical solution.
The periodic boundary condition is:

$\psi(0, t)=\psi(L, t)$,

where $L$ is the size of integration area (we shall use $L=3 \cdot 10^{7} \mathrm{~m}$ ). The solution of Eq. (6) can be written as

$\psi(x, t)=\sum_{n=1}^{N} A_{n} \sin \left[k_{n}\left(x-c_{n} t\right)+\phi_{n}\right]$,

where

$c_{n}=U-\frac{\beta+U / l_{0}^{2}}{k_{n}^{2}+1 / l_{0}^{2}}$,

$k_{n}=\frac{2 \pi n}{L}$,

and $A_{n}$ and $\phi_{n}$ are defined by the initial condition (Rossby, 1939).

For finding the numerical solution it is convenient to rewrite the equation in nondimensional form. We choose the following scales: $S=6 \cdot 10^{6} \mathrm{~m}, T=S / V=6 \cdot 10^{5} \mathrm{~s}, V=10 \mathrm{~m} / \mathrm{s}$. The dependent and independent nondimensional variables are defined as follows:

$\tilde{x}=\frac{x}{S}, \quad \tilde{t}=\frac{t}{T}, \quad \tilde{\psi}=\frac{T}{S^{2}} \psi$.

Equation (6) may be written in nondimensional form as

$\frac{\partial}{\partial \tilde{t}}\left(\frac{\partial^{2}}{\partial \tilde{x}^{2}}-\frac{1}{b^{2}}\right) \tilde{\psi}+\beta_{0} \frac{\partial \tilde{\psi}}{\partial \tilde{x}}+U_{0} \frac{\partial^{3} \tilde{\psi}}{\partial \tilde{x}^{3}}=0$,

where $\frac{1}{b}=\frac{S}{l_{0}}=2, \beta_{0}=\frac{\beta S^{2}}{V}=57.6$ and $U_{0}=\frac{U}{V} \in[0,3]$.

For finite-difference discretization we use the unconditionally stable scheme with truncation error $\mathcal{O}\left(\Delta x^{2}, \Delta t^{2}\right)$ given by

$$
\begin{aligned}
& \frac{1}{\Delta t}\left(\frac{\tilde{\psi}_{i+1}^{k+1}-2 \tilde{\psi}_{i}^{k+1}+\tilde{\psi}_{i-1}^{k+1}}{\Delta x^{2}}-\frac{\tilde{\psi}_{i+1}^{k}-2 \tilde{\psi}_{i}^{k}+\tilde{\psi}_{i-1}^{k}}{\Delta x^{2}}-\right. \\
& \left.\frac{1}{b^{2}}\left(\tilde{\psi}_{i}^{k+1}-\tilde{\psi}_{i}^{k}\right)\right)+\frac{\beta_{0}}{2}\left(\frac{\tilde{\psi}_{i+1}^{k+1}-\tilde{\psi}_{i-1}^{k+1}}{2 \Delta x}+\frac{\tilde{\psi}_{i+1}^{k}-\tilde{\psi}_{i-1}^{k}}{2 \Delta x}\right)+ \\
& \frac{U_{0}}{2}\left(\frac{\tilde{\psi}_{i+2}^{k+1}-2 \tilde{\psi}_{i+1}^{k+1}+2 \tilde{\psi}_{i-1}^{k+1}-\tilde{\psi}_{i-2}^{k+1}}{2 \Delta x^{3}}+\right. \\
& \left.\frac{\tilde{\psi}_{i+2}^{k}-2 \tilde{\psi}_{i+1}^{k}+2 \tilde{\psi}_{i-1}^{k}-\tilde{\psi}_{i-2}^{k}}{2 \Delta x^{3}}\right)=0
\end{aligned}
$$

At first, we generate a specific analytical solution (8) containing 85 modes. Figure 5 shows this solution for $t=0$.

Note that in the figures we use dimensional values, but in the numerical computations all variables are nondimensional. 


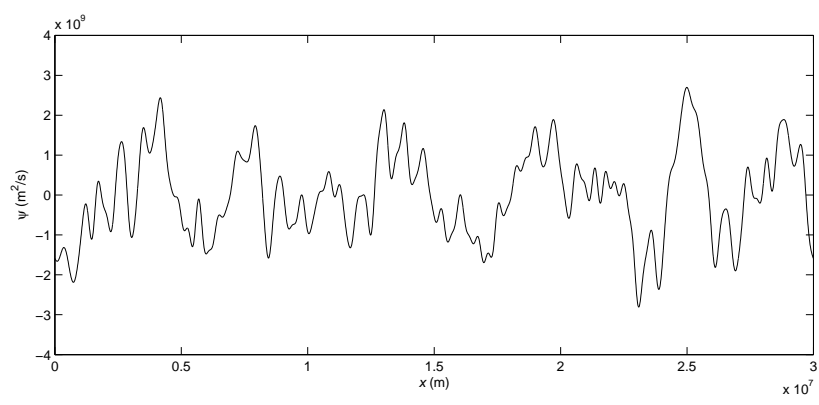

Fig. 5. Analytical solution of Rossby-Oboukhov equation on periodic domain $[0, L]$ at $t=0 \mathrm{~h}$ with 85 modes.

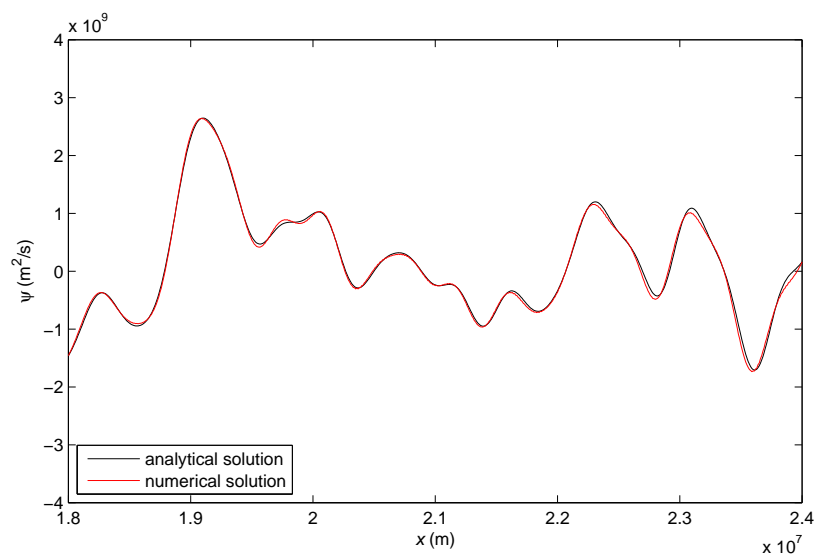

Fig. 6. Analytical (black line) and numerical (red line) CauchyDirichlet problem solution of Rossby-Oboukhov equation, with exact boundary condition, at $t=96 \mathrm{~h} . \Delta x=10 \mathrm{~km}, \Delta t=200 \mathrm{~s}$. CPU time $=0.8 \mathrm{~s}$.

As a local model we consider the Eq. (11), with initial and boundary conditions, defined over $[a, b] \mp[0, L]$ (closed interval smaller than the entire domain). For initial and boundary conditions we will use our specific form (see Fig. 5) of global model solution (8). In order to be closer to real problems of atmospheric modelling we take the analytical solution in the points of the coarse grid with space step $\Delta x=200 \mathrm{~km}$ and time step $\Delta t=2 \mathrm{~h}$, and perturb its values randomly in such a way that a perturbation can reach till $30 \%$ of its exact value.

Primarily we find the solution of the Cauchy-Dirichlet problem for our local model. As local domain we take the interval $\left[1.8 \cdot 10^{7}, 2.4 \cdot 10^{7}\right] \mathrm{m}(6000 \mathrm{~km}$ of length) inside the global model interval $[0, L]$. For the first reference experiment the initial and boundary conditions were taken from the exact analytical solution (8). Requiring that the numerical solution with the exact initial and boundary condition have to nearly coincide with the analytical solu
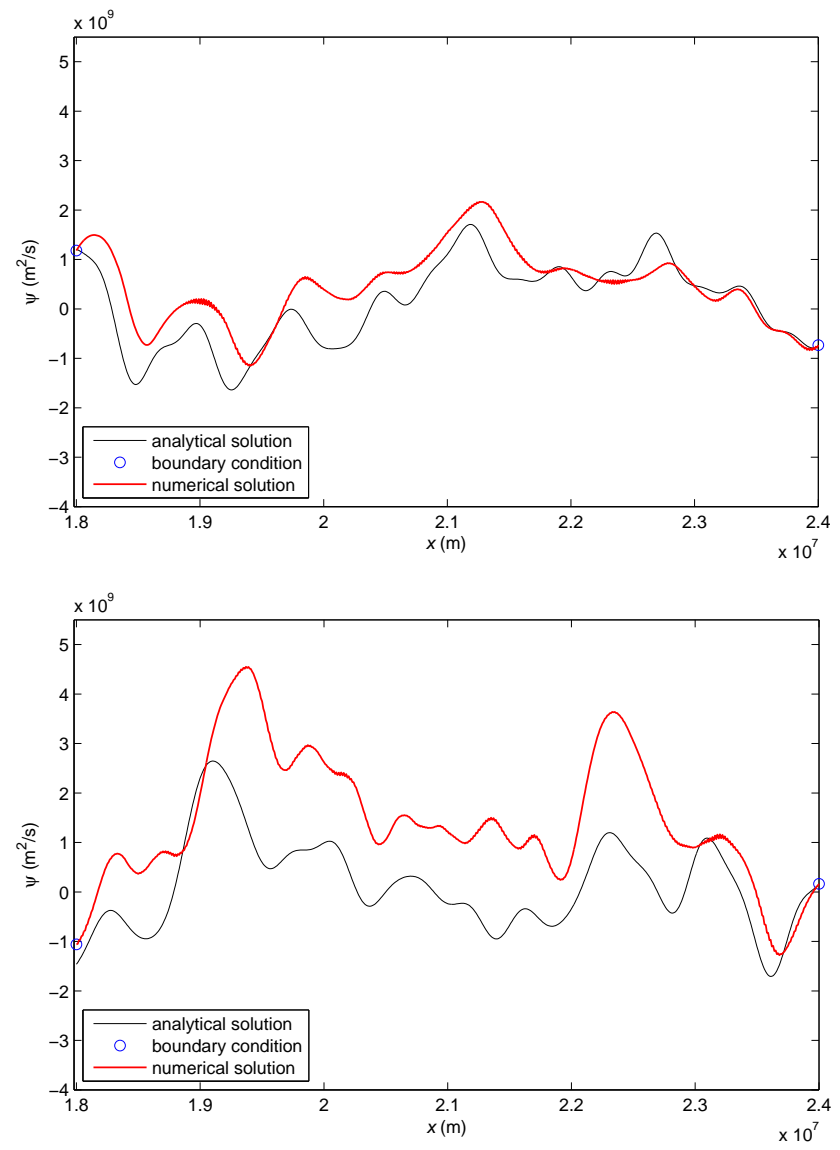

Fig. 7. Numerical Cauchy-Dirichlet problem solution of RossbyOboukhov equation (red line) with boundary condition obtained from $30 \%$ perturbed global model values (circles) at $t=48 \mathrm{~h}$ (upper panel), and at $t=96 \mathrm{~h}$ (lower panel). $\Delta x=10 \mathrm{~km}, \Delta t=200 \mathrm{~s}$. CPU time $=0.8 \mathrm{~s}$. Black line corresponds to the reference analytical reference solution.

tion,we choose as the maximal possible values of the space and time steps, $\Delta x=10 \mathrm{~km}$ and $\Delta t=200 \mathrm{~s}$, respectively. To obtain the numerical solution for $96 \mathrm{~h}$, showed in Fig. 6 , it is demanded $0.8 \mathrm{~s}$ of CPU time.

In the next experiments, Fig. 7, the perturbed analytical solution on the coarse grid was used for formation of the boundary conditions.

One can see that for the Cauchy-Dirichlet problem the numerical solution rather quickly diverges from the analytical solution due to the errors of the linear interpolation procedure in the border points. After $96 \mathrm{~h}$, the perturbed boundary solution has very faint resemblance with the analytical one.

In the second series of experiments we applied the optimization method to the local model. For this calculation we used all available global data (perturbed analytical solution on coarse grid) in the inner local domain. Figure 8 shows the results of calculations. It is important to note that, as in previous experiments, we chose the steps in the space $\Delta x=100 \mathrm{~km}$ and in the time $\Delta t=3600 \mathrm{~s}$ to be as large as possible. 

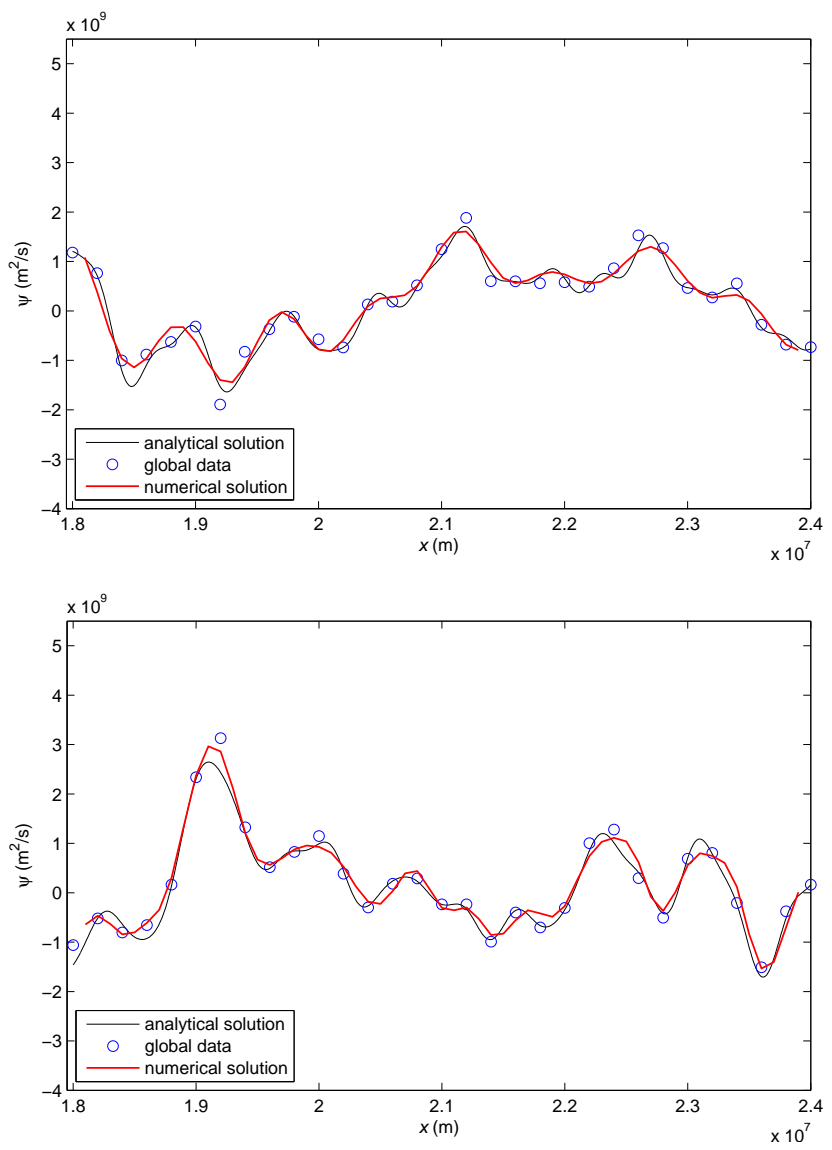

Fig. 8. Numerical solution of Rossby-Oboukhov equation by optimization problem (red line) using $30 \%$ perturbed global model values (circles) at $t=48 \mathrm{~h}$ (upper panel), and at $96 \mathrm{~h}$ (lower panel). $\Delta x=100 \mathrm{~km}, \Delta t=3600 \mathrm{~s}$. CPU time $=0.5 \mathrm{~s}$. Black line corresponds to the reference analytical solution.

In the optimization approach one can use larger steps than in the Cauchy-Dirichlet problem, because we do not deal with a time-evolution problem, and there is no accumulation of numerical errors at each time step. In Fig. 8, one can see that, for space and time steps many times larger than those that were used in the Cauchy-Dirichlet problem $(\Delta x=10 \mathrm{~km}$ and $\Delta t=200 \mathrm{~s}$ ), we have far better agreement with the analytical solution. The decrease of time and/or space step in the optimization approach does not appreciably improve the solution. Only the smallest details are slightly better reproduced.

Since the local mesh in the optimization approach is rather coarse, the average CPU time needed for solving the problem is smaller than for Cauchy-Dirichlet problem $(0.5 \mathrm{~s})$ in spite of greater computational complexity. Also we have to pay attention on a very weak sensitivity to the global data perturbations. The impact of earch individual perturbation on the optimization solution is very small. For example, increasing errors in randomly perturbed global data up to $60 \%$ does not strongly affect the solution as one can see in Fig. 9.

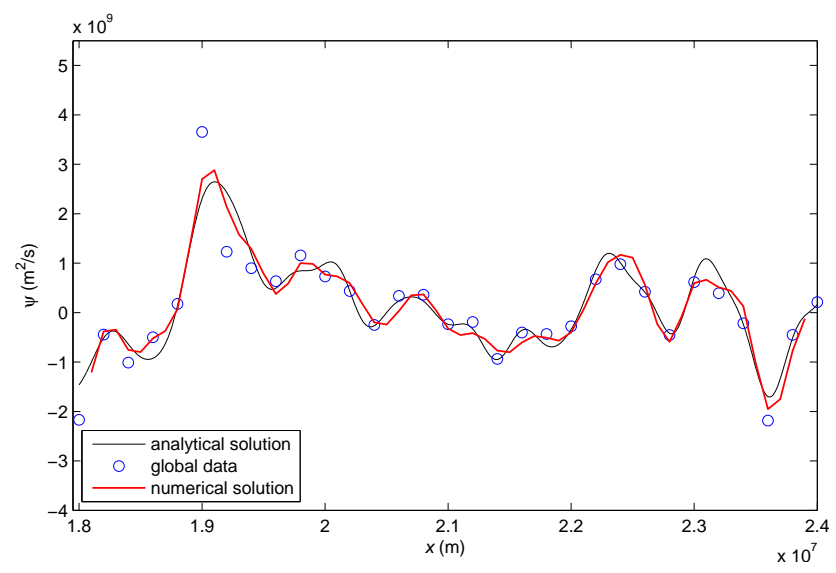

Fig. 9. Numerical solution of Rossby-Oboukhov equation by optimization problem (red line) with $60 \%$ perturbed global model values (circles) at $t=96 \mathrm{~h} . \Delta x=100 \mathrm{~km}, \Delta t=3600 \mathrm{~s}$. CPU time $=0.5 \mathrm{~s}$. Black line corresponds to the reference analytical solution.

With the above experiments we had the intention to show that even small errors in boundary conditions of the regional model can violently distort ideal solution (i.e., the one that is obtained when there are no errors in boundary conditions) However, applying optimization methods, and using the global data (from observation or from "global" model) available inside the regional domain (even containing the same kind of errors as the boundary conditions), gives us a way to avoid strong sensitivity of solution to boundary errors, allowing thus to obtain good results.

In our experiments the regional model has not the ability to add small scale spectrum to the global model solution, because we use the same evolution model operator for both models. So, to simulate more realistic behavior let us modify the testing regional model.

First we generate more realistic global data omiting small scales. Using as the initial condition only the first 25 modes (the whole solution includes 85 modes) taken from analitical solution, showed on Fig. 5, we run the model with space and time steps equal to $100 \mathrm{~km}$ and $1800 \mathrm{~s}$, respectively, on the entire periodic domain of $96 \mathrm{~h}$. After that we extract from the obtained solution the global data, on the mesh with size $200 \mathrm{~km}$ and $2 \mathrm{~h}$.

Note that the first harmonics in Rossby-Obukhov equation solution (8) have the negative velocity, that is, the largest scale Rossby waves are westward moving and carry signal from the right boundary to the left one. All small scales are traveling eastward (from the left to the right in our figures). Therefore, the boundary condition for the modified regional model is derived from global data, but additionaly, at the left boundary, the small scales from analytical solution that are not present on global solution are introduced (at the points of the global mesh). The resulted model attempts to reproduce the small scales in the system, but there is the error on the 

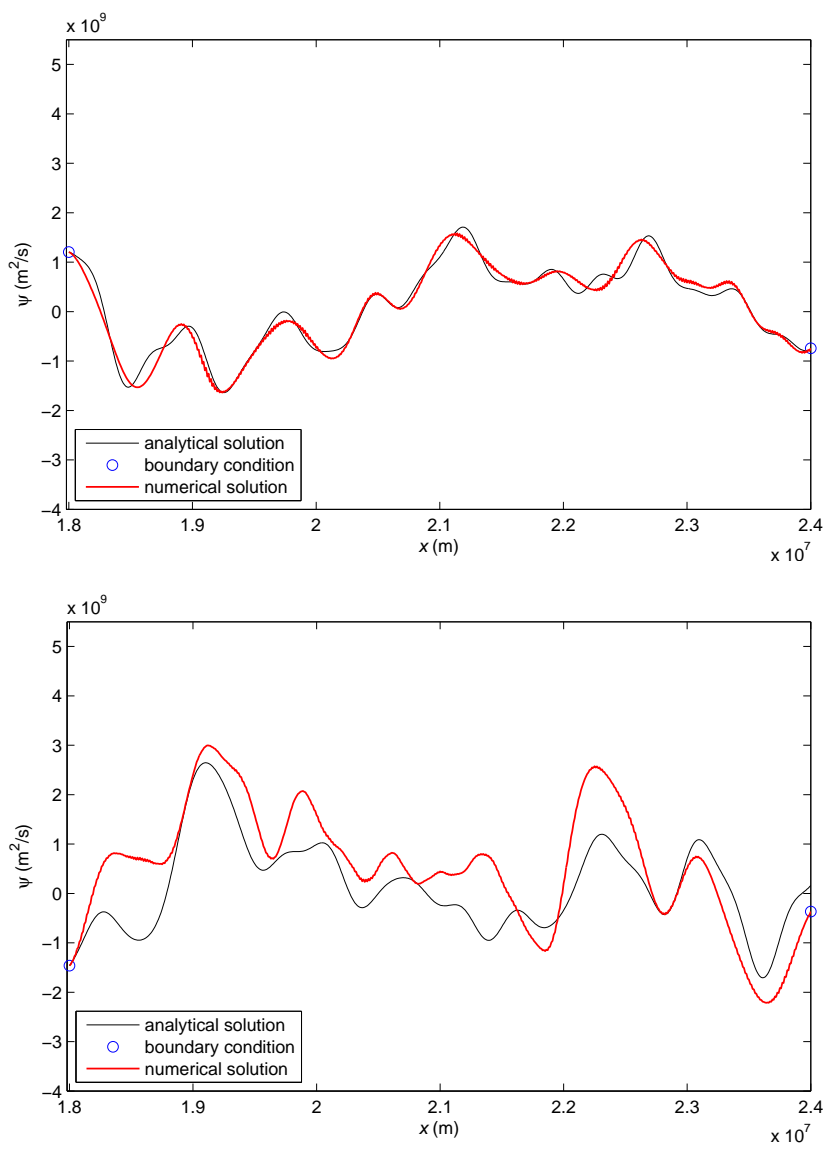

Fig. 10. Numerical Cauchy-Dirichlet problem solution of RossbyOboukhov equation (red line) with small scales introduced in the left side of boundary conditions (circles) at $t=48 \mathrm{~h}$ (upper panel) and $96 \mathrm{~h}$ (lower panel). $\Delta x=10 \mathrm{~km}, \Delta t=200 \mathrm{~s}$. CPU time $=0.8 \mathrm{~s}$. Black line corresponds to the reference analytical solution.

boundary due to interpolation. Using new regional model and global data without small scales we performed other experiments using traditional and optimization approaches, the results of which are showed in Figs. 10 and 11, respectively.

These experiments clearly show that the use of additional global data inside the local domain, even perturbed ones, can significantly improve the solution of the model.

\subsection{Korteweg-de Vries equation}

The Korteweg-de Vries (KdV) equation is a nonlinear, dispersive partial differential equation and represents a mathematical model of waves on shallow water surfaces:

$u_{t}+6 u u_{x}+u_{x x x}=0$.
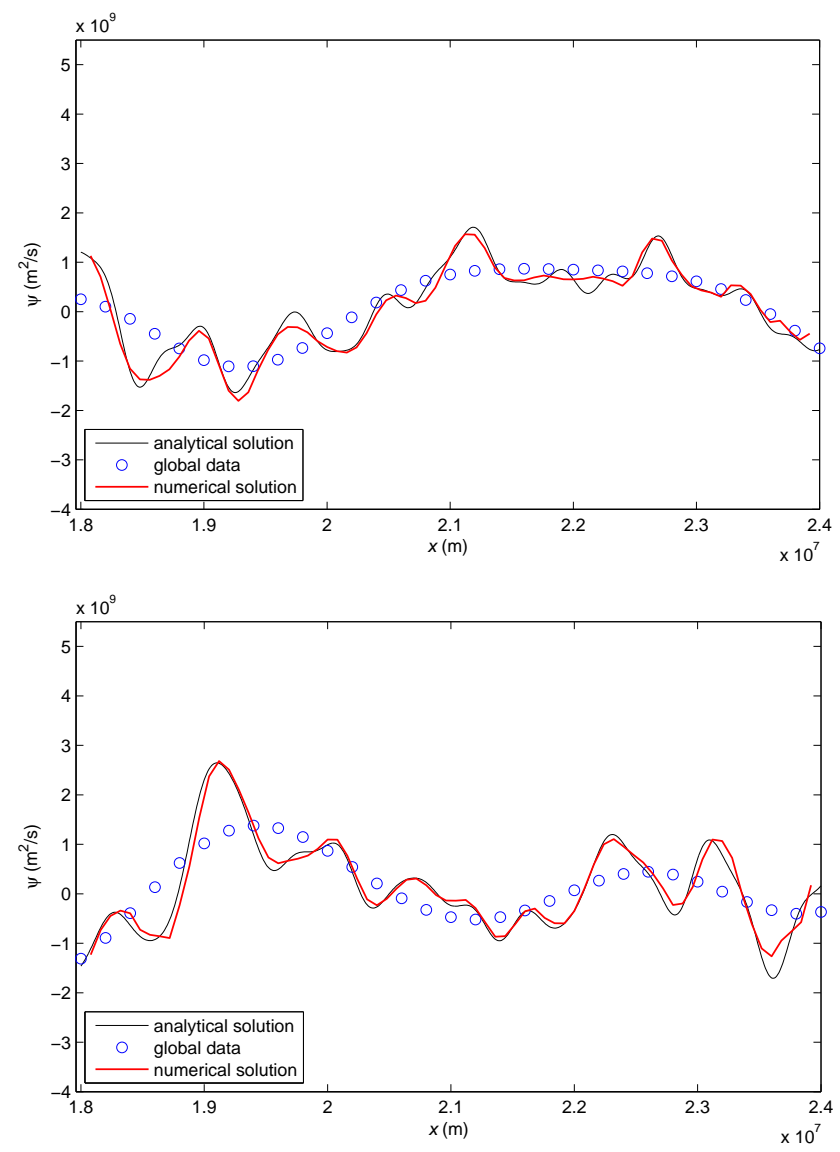

Fig. 11. Numerical solution of Rossby-Oboukhov equation by optimization problem (red line), using as global data (circles) the global model solution with 25 modes and additional small scales in the left boundary, at $t=48 \mathrm{~h}$ (upper painel) and at $96 \mathrm{~h}$ (lower painel). $\Delta x=80 \mathrm{~km}, \Delta t=2600 \mathrm{~s}$. CPU time $=1.25 \mathrm{~s}$. Black line corresponds to the reference analytical solution.

Equation (12) has the exact solution (Korteweg and de Vries, 1895; Grimshaw, 2004)

$u(x, t)=b+a \mathrm{cn}^{2}(\gamma(x-V t) \mid m)$,

where $\operatorname{cn}(x \mid m)$ is the Jacobi elliptic function, $m \in(0,1)$ is the module of elliptic function, $a=2 m \gamma^{2}$ and $V=6 b+4(2 m-1) \gamma^{2}$. For the case when $m \rightarrow 1$ we will have $\operatorname{cn}(x \mid m) \rightarrow \operatorname{sech}(x)$ and the solution (13) will have the form

$u(x, t)=b+a \operatorname{sech}^{2}(\gamma(x-V t))$,

with $V=6 b+2 a$, and $a=2 \gamma^{2}$ which describes a onedimensional soliton. 


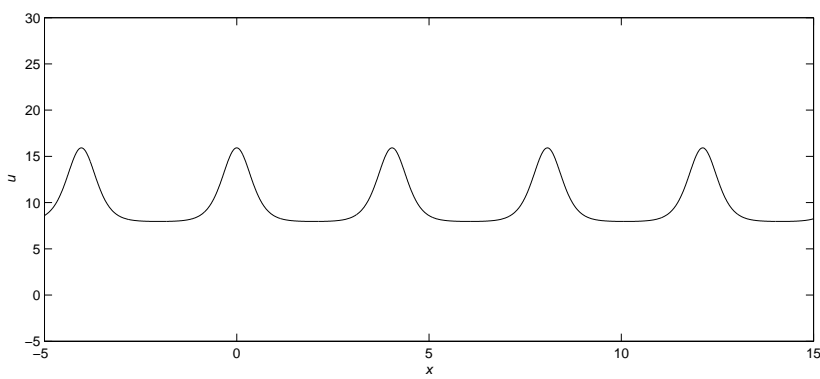

Fig. 12. Analytical solution of $\mathrm{KdV}$ equation as the cnoidal wave (13) on the domain $[-5,15]$ at $t=0$ with $\gamma=2, b=a$ and module $m=0.995$.

For finite-difference discretization we use the following implicit numerical scheme (Furihata, 1999), which possesses the properties of total energy and mass conservation:

$$
\begin{aligned}
& \frac{u_{i}^{j+1}-u_{i}^{j}}{\Delta t}+\frac{1}{2 \Delta x}\left(\left(u_{i+1}^{j+1}\right)^{2}-\left(u_{i-1}^{j+1}\right)^{2}+u_{i+1}^{j+1} u_{i+1}^{j}-\right. \\
& \left.u_{i-1}^{j+1} u_{i-1}^{j}+\left(u_{i+1}^{j}\right)^{2}-\left(u_{i-1}^{j}\right)^{2}\right)+ \\
& \frac{1}{2 \Delta x^{3}}\left(\frac{u_{i+2}^{j+1}+u_{i+2}^{j}}{2}-\left(u_{i+1}^{j+1}+u_{i+1}^{j}\right)+\right. \\
& \left.\left(u_{i-1}^{j+1}+u_{i-1}^{j}\right)-\frac{u_{i-2}^{j+1}+u_{i-2}^{j}}{2}\right)=0 .
\end{aligned}
$$

Since the numerical scheme is non-linear for numerical evaluation of Cauchy-Dirichlet problem we use Newton's method for computing $u^{j+1}$ at each time step.

Following the steps of previous example, we choose as a reference model the analytical solution with cofficients $b=a$, $\gamma=2$ and module of elliptic function $m=0.995$. Figure 12 represents the solution on the domain $[-5,15]$ at time $t=0$.

As a local model we consider the Eq. (12) defined over the closed interval $[0,10]$. In order to get a good accordance between a numerical solution of the Cauchy-Dirichlet problem (with exact initial and boundary conditions) and the analytical solution on the time interval $0 \leq t \leq 1$, showed in Fig. 13, we take as the maximum possible values of space and the time steps $\Delta x=0.02$ and $\Delta t=0.0002$, respectively. The CPU time required to find the solution is $15 \mathrm{~s}$.

As a global model solution we take the analytical solution, introduced above as the reference model, with space step $\Delta x=0.5$ and time step $\Delta t=0.005$ and perturb its values up to $10 \%$ from the exact ones. Using these perturbed global model solution to form the boundary condition we solve the Cauchy-Dirichlet problem.

In Fig. 14 one can see that the Cauchy-Dirichlet problem with small errors in the boundary conditions can produce unacceptable numerical solutions.

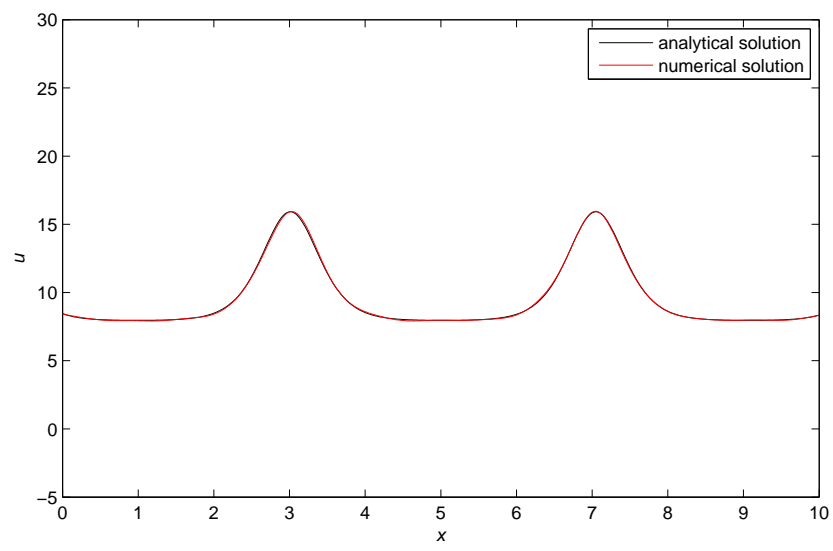

Fig. 13. Numerical Cauchy-Dirichlet problem solution of $\mathrm{KdV}$ equation with exact boundary condition (red line) at $t=1 . \Delta x=0.02$, $\Delta t=0.0002$. CPU time $=15 \mathrm{~s}$. Black line corresponds to the reference analytical solution.
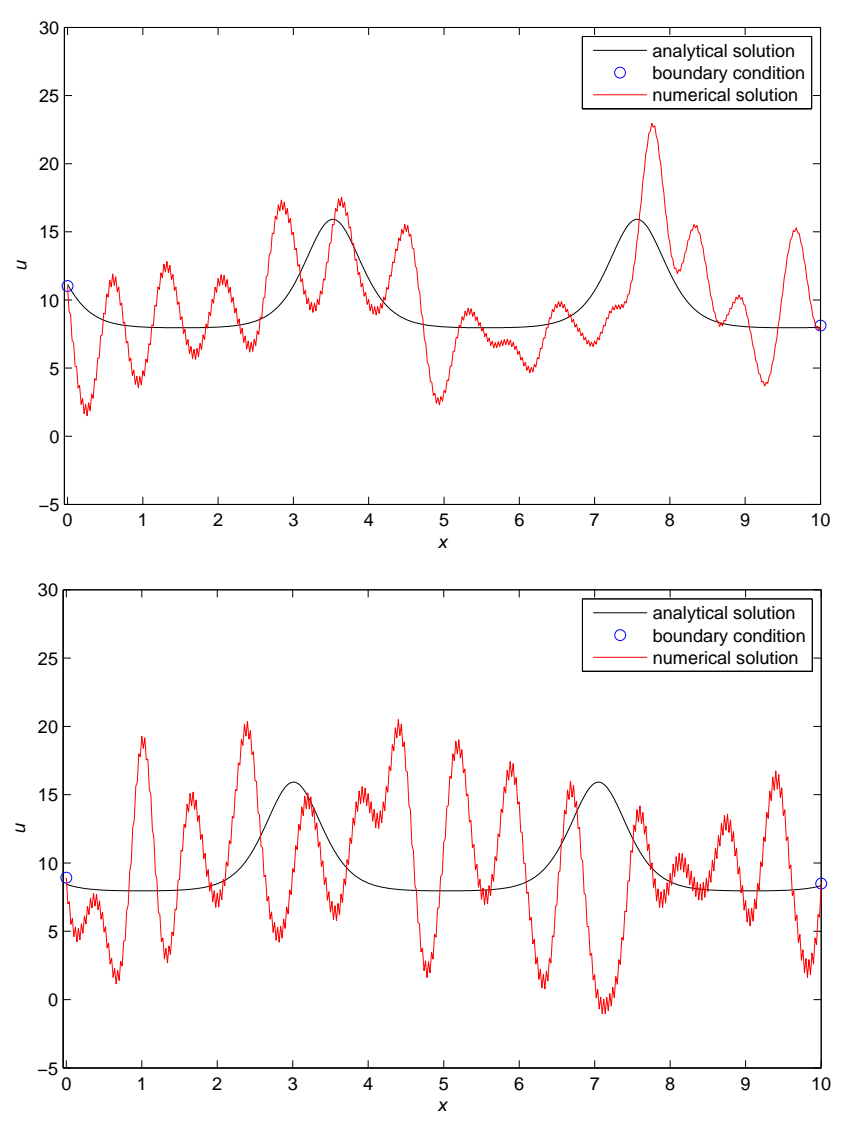

Fig. 14. Numerical Cauchy-Dirichlet problem solution of KdV equation (red line) with $10 \%$ perturbed boundary condition (circles) at time $t=0.5$ (upper panel), and $t=1.0$ (lower panel). $\Delta x=0.02$, $\Delta t=0.0002$. CPU time $=23 \mathrm{~s}$. Black line corresponds to the reference analytical solution. 
Now, we apply the optimization method to the local model using all the avaliable points of the global model solution. Figure 15 shows the solution of the optimization problem. As in the previous experiment we choose the space $(\Delta x=0.2)$ and the time $(\Delta t=0.002)$ steps as large as possible.

It can be clearly seen the advantage of the optimization approach in this case. The difference between numerical and exact solution is very small in comparison with the CauchyDirichlet problem calculations.

\section{Conclusions and discussions}

The results of the numerical experiments presented here indicate that the optimization approach can significantly improve the precision of the numerical solution of the regional model when the boundary values have errors but information in a number of inner points is available. Even in the cases in which the solution of the Cauchy-Dirichlet problem is very sensitive to the errors in the boundary condition, the use of optimization approach gives the possibility to compute solutions that are close to the analytical solution. We also made experiments with two-dimensonal nonlinear RossbyOboukhov equations. The preliminary results demonstrate as well that the use of the optimization approach significantly improve the numerical solution.

Applying 4D-Var data assimilation to limited-area problem has some common features with the optimization approach used here. In a series of works devoted to optimal control of initial and lateral boundary conditions it is emphasized the strong sensitivity of obtained solution to the lateral baundary data (Griffin and Thomson, 1996; Lu and Browning, 2000; Zou and Kuo, 1996; Leredde et al., 1998). As it was shown in the above mentioned papers, the consistency of lateral boundary conditions with another "observation" data permit to obtain more realistic regional model fields. In our approach this consistency between "regional model" and "global model" is assured automatically by optimization solution procedure for the whole solution period.

In connection with applying a deterministic 4D-Var scheme to find the most likely model evolution fitting observations, (Lorenc and Payne, 2007) pointed out that it does not work in the limit of high resolution. On the other hand, Statistical 4D-Var (Lorenc, 2003) is a suitable alternative. The reason is that deterministic $4 \mathrm{D}$-Var aims to find the most probable solution, which corresponds to the mode of the probability distribution function whereas statistical 4D-Var seeks an efficient approximation to the probability density function expectation. Let us interpret the observations of Lorenc and Payne in optimization terms and see how they apply to our case. For simplicity, the independent variables are denoted by $x$ so that our problem is to minimize $f(x)$ (the error) subject to $x \in \Omega$ (the model). The mode solution corresponds to the global minimizer of $f$. The choice of the global minimizer as the best solution may be challenged if the basin
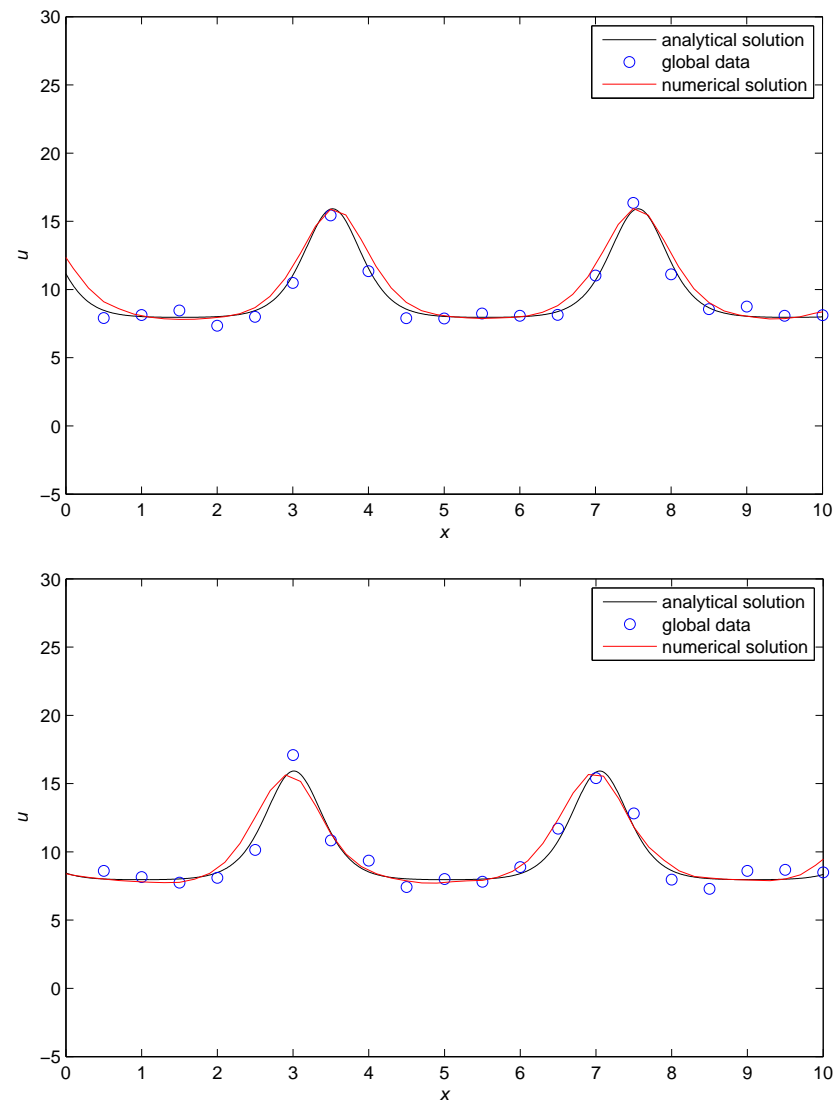

Fig. 15. Numerical solution of KdV equation by optimization problem (red line) with $10 \%$ perturbed global data (circles) at time $t=0.5$ (upper panel), and at $t=1.0$ (lower panel). $\Delta x=0.2, \Delta t=0.002$. CPU time $=21 \mathrm{~s}$. Black line corresponds to the reference analytical solution.

of attraction of it is very narrow, so that relatively small perturbations of $x$ may produce large error increasing. Clearly, a merely local solution with a larger attraction basin would be much better as a model approximation, since their perturbations would preserve a reasonably moderate error. Such a local solution roughly corresponds to the maximization of expectancy introduced by Lorenc and endorsed in (Lorenc and Payne, 2007). The obvious way to approximate the optimal expectancy relies on modifying the optimization problem so that the desired estimation would be a global minimizer of the new problem. Filtering techniques are the proper tool for this purpose. Essentially, the new modified problem will be to minimize $F(f(x))$ instead of minimizing $f(x)$, where the operator $F$ represents a (convolution) filter. This procedure tends to stabilize the optimization problem, so that jumps between unstable global minimizers and perhaps stable suitable local minimizers do not occur. Moreover, a high-frequency variation of $x$ is less prone to disappear, because we seek the approximation of smoothed version of $x$ to the global (smoother) model, instead of an approximation of $x$ itself. 


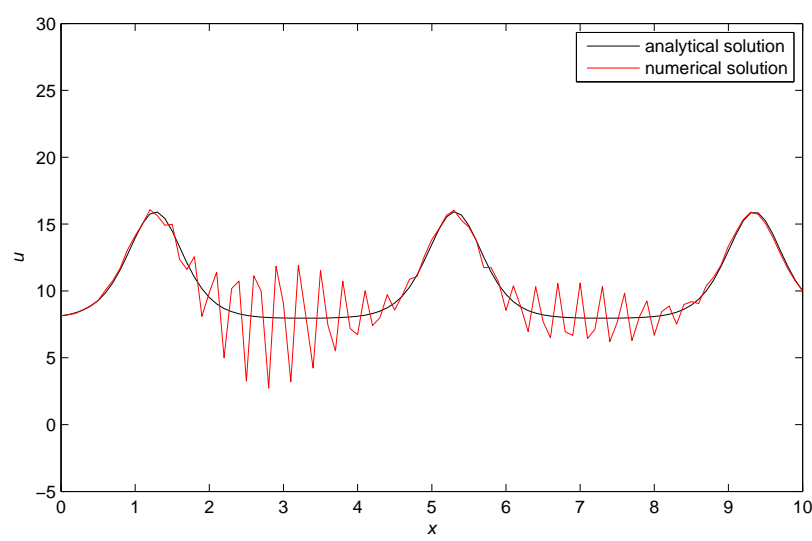

Fig. A1. Numerical evolutionary (Cauchy-Dirichlet problem) solution of KdV equation using center-space and center-time scheme (red line) after 40 steps with $\Delta x=0.1$ and $\Delta t=5 * 10^{-4}$. Black line corresponds to the reference analytical solution.

\section{Appendix A}

\section{Formulation of optimization problem}

We can express our problem as the following non-linear optimization problem with equality constraints

Minimize $\frac{1}{2}\|u-V\|_{P}^{2}$

where $V$ represents the global data on the regional mesh, $P$ is the diagonal penality matrix and $h(u)=\left[h_{1}(u) h_{2}(u) \ldots h_{m}(u)\right]^{T}$ is a vector of the discretizations of regional equations at each point of space-time mesh of the regional model.

A usual optimization technique to solve this kind of problem is to apply Newton's iteration method to the system of nonlinear equations arising from first-order necessary conditions, known as Karush-Kuhn-Tucker (KKT) conditions (Nocedal and Wright, 1999). For instance, the KKT conditions for the problem (A1) are

$$
\begin{aligned}
P(u-V)+h^{\prime}(u)^{T} \lambda & =0 \\
h(u) & =0,
\end{aligned}
$$

where

$h^{\prime}(u)=\left(\begin{array}{c}\nabla h_{1}(u)^{T} \\ \nabla h_{2}(u)^{T} \\ \vdots \\ \nabla h_{m}(u)^{T}\end{array}\right)$

is the Jacobian matrix of the constraint function and $\lambda$ represent the vector of Lagrange multipliers. Each step of the Newton iteration associated with system (A2) is defined as follows:

$$
\begin{aligned}
J\left(u_{k}, \lambda_{k}\right)\left(\begin{array}{l}
\Delta u_{k} \\
\Delta \lambda_{k}
\end{array}\right) & =-\left(\begin{array}{l}
P\left(u_{k}-V\right)+h^{\prime}\left(u_{k}\right)^{T} \lambda_{k} \\
h\left(u_{k}\right)
\end{array}\right) \\
\left(\begin{array}{l}
u_{k+1} \\
\lambda_{k+1}
\end{array}\right) & =\left(\begin{array}{l}
u_{k} \\
\lambda_{k}
\end{array}\right)+\left(\begin{array}{c}
\Delta u_{k} \\
\Delta \lambda_{k}
\end{array}\right)
\end{aligned}
$$

where $J$ represents the Jacobian matrix of the system (A2)

$J(u, \lambda)=\left(\begin{array}{cc}P+\sum_{i} \lambda_{i} \nabla^{2} h_{i}(u) & h^{\prime}(u)^{T} \\ h^{\prime}(u) & 0\end{array}\right)$,

and $\nabla^{2} h_{i}(u), i=1, \ldots, m$, are Hessian matrices of the constraints.

The Jacobian matrix (A4) is a saddle point matrix and there are many methods that can be applied for the associated linear system (Benzi, Golub and Liesen, 2005). However, note that the Jacobian of the constraints $h^{\prime}(u)$ is described by a sparse matrix with block-diagonal structure. On the other hand, the first part $P+\sum_{i} \lambda_{i} \nabla^{2} h_{i}(u)$ of the Jacobian matrix (A4) of the system (A2) includes the calculation of the Hessians of the constraints, which is computationally expensive because calculations have to be made for every Newton iteration. The resulting matrix is generally dense. To accelerate the calculations and reduce the computer memory requirements we use the following Jacobian approximation:

$B_{k}=\left(\begin{array}{cc}P & h^{\prime}\left(u_{k}\right)^{T} \\ h^{\prime}\left(u_{k}\right) & 0\end{array}\right)$.

The use of the approximation $B_{k}$ for $J\left(u_{k}, \lambda_{k}\right)$ strongly simplifies the procedure of finding the solution of the linear system (A3).

\section{Appendix B}

\section{Mesh size on optimization approach}

To describe the same space and time scales, the optimization approach can use larger space and time steps than the traditional evolutionary approach. The key to explain this phenomenon is that the optimization algorithm is nonevolutionary in time, hence it is not necessary to satisfy the stability condition. Moreover, there is no accumulation of discretization error. Therefore, for any period of integration, space and time steps depend only on the scales of the process of interest.

The stability condition of numerical schemes is given by a relationship between space and time steps that guarantees the stability during the evolution. Frequently, this relation is very strong. For example, using a simple center-space and 


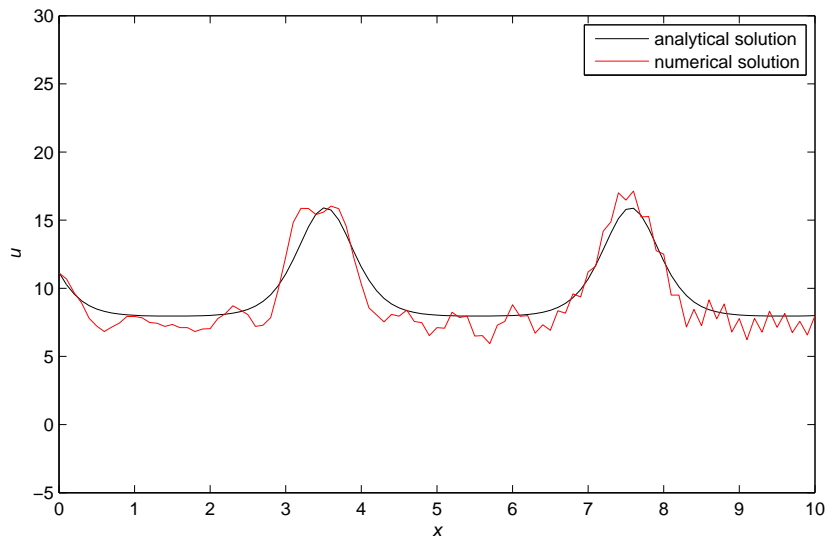

Fig. B1. Numerical evolutionary (Cauchy-Dirichlet problem) solution of $\mathrm{KdV}$ equation using center-space and center-time scheme (red line) at time $t=0.5$ with $\Delta x=0.1$ and $\Delta t=4.5 * 10^{-4}$. Black line corresponds to the reference analytical solution.

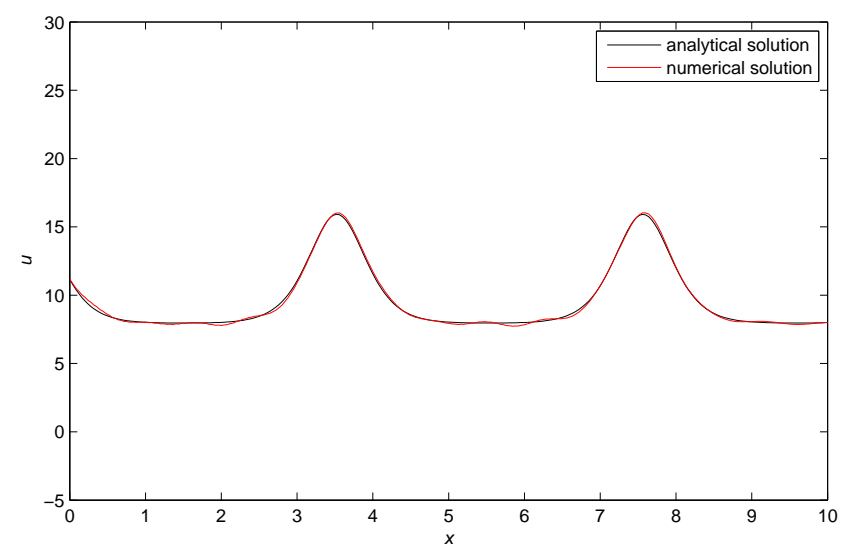

Fig. B2. Numerical evolutionary (Cauchy-Dirichlet problem) solution of $\mathrm{KdV}$ equation using center-space and center-time scheme (red line) at time $t=0.5$ with $\Delta x=0.04$ and $\Delta t=2 * 10^{-5}$. Black line corresponds to the reference analytical solution.

center-time scheme for discretization of the KdV equation

$$
\begin{aligned}
\frac{u_{i}^{k+1}-u_{i}^{k-1}}{2 \Delta t}+ & 6 u_{i}^{k} \frac{u_{i+1}^{k}-u_{i-1}^{k}}{2 \Delta x}+ \\
& \frac{u_{i+2}^{k}-2 u_{i+1}^{k}+2 u_{i-1}^{k}-u_{i-2}^{k}}{2 \Delta x^{3}}=0
\end{aligned}
$$

and taking the space step $\Delta x=0.1$, we obtain the stable solution, by traditional approach, only for time steps $\Delta t \leq 4.5 *$ $10^{-4}$. Figure A1 shows the instability of scheme for time step $\Delta t=5 * 10^{-4}$ after 40 steps.

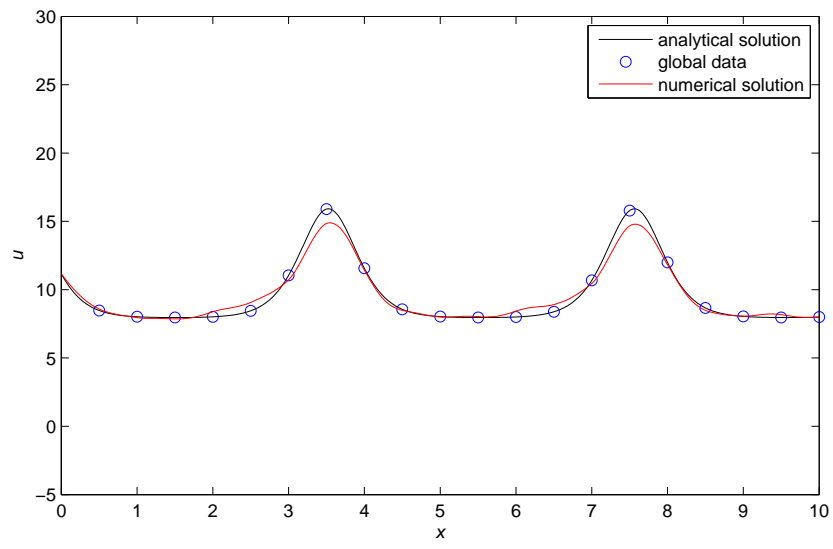

Fig. B3. Numerical optimization solution of KdV equation using center-space and center-time scheme (red line) at time $t=0.5$ with $\Delta x=0.04$ and $\Delta t=2.5 * 10^{-3}$. Black line corresponds to the reference analytical solution, circles marks global data.

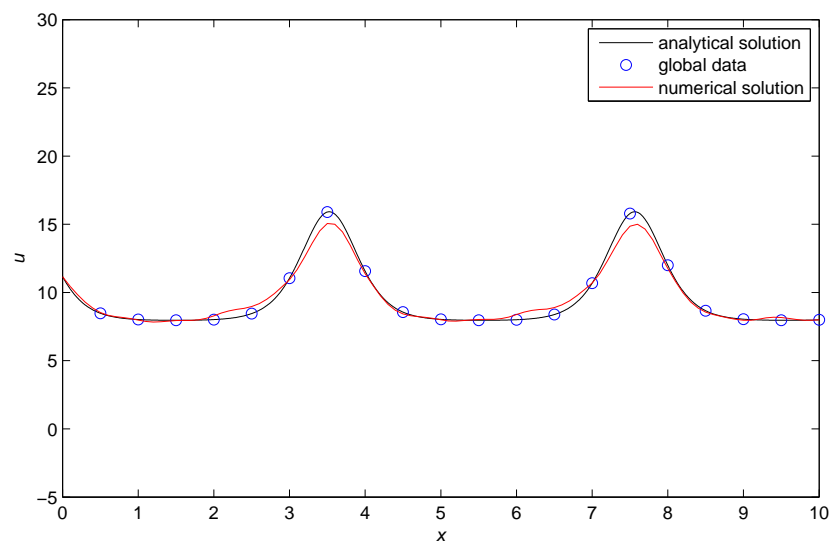

Fig. B4. Numerical optimization solution of KdV equation using center-space and center-time scheme (red line) at time $t=0.5$ with $\Delta x=0.1$ and $\Delta t=2.5 * 10^{-3}$. Black line corresponds to the reference analytical solution, circles marks global data.

In addition to this, for long time evolutions the accumulation of discretization error tends to be more significant. As we can see in Fig. B1, for time period 0.5 space step $\Delta x=0.1$ and time step $\Delta t \leq 4.5 * 10^{-4}$ produce a bad result. So it is necessary to refine the mesh when the period of integration becomes longer. In the case of $t=0.5$ a resonable result is achieved for $\Delta x=0.04$ and $\Delta t=2 * 10^{-5}$ (see Fig. B2).

Keeping the same space step and taking the global data as the exact solution on the mesh with space-time size equals to $0.5 \times 0.01$, the optimization approach converges to the solution with the time step $\Delta t=2.5 * 10^{-3}$ (Fig. B3). Note here that the number of global points on the space $\times$ time domain with resolution $[0,10] \times[0,0.5]$ in the optimization problem is equal to 1000 , whereas the number of boundary condition points in the traditional approach with $\Delta t=2 * 10^{-5}$ is $10^{5}$. 
As we can see, for the optimization approach there is no the same concept of stability as for the forward problem. Besides allowing a larger time step, the optimization approach also allows the use of a larger space step, since there is no accumulation of discretization error, as we mentioned above. Figure B4 shows the optimization solution for $\Delta x=0.1$ and $\Delta t=2.5 * 10^{-3}$

Acknowledgements. The authors gratefully acknowledge R. Laprise for constructive discussions which helped to make clear a number of difficulties of the problem, and T. A. Tarasova for useful comments on the text that significantly improved the manuscript. The authors also express great gratitude to editor O. Talagrand and two referees for valuable remarks and useful references.

F. I. Pisnitchenko was supported by FAPESP (Grant 03-09938-0); J. M. Martínez was supported by FAPESP (Grant 06-53768-0) and $\mathrm{CNPq}$ and S. A. Santos was supported by CNPq.

Edited by: O. Talagrand

Reviewed by: A. Lorenc and another anonymous referee

\section{References}

Andreani, R., Birgin, E. G., Martínez, J. M., and Schuverdt, M. L.: On Augmented Lagrangian methods with general lower-level constraints, SIAM J. Optimiz., 18, 1286-1309, 2007.

Andreani, R., Birgin, E. G., Martínez, J. M., and Schuverdt, M. L.: Augmented Lagrangian methods under the Constant Positive Linear Dependence constraint qualification, Math. Program., 111, 5-32, 2008.

Benzi, M., Golub, G. H., and Liesen, J.: Numerical Solution of Saddle Point Problems, Acta Numerica, 1-137, Cambridge University Press, 2005.

Birgin, E. G. and Evtushenko, Y.: Automatic differentiation and spectral projected gradient methods for optimal control problems, Optimization methods and software, 10, 20-42, 1998.

Birgin, E. G. and Martínez, J. M.: Improving ultimate convergence of an Augmented Lagrangian method, Optimization Methods and Software, 23, 177-195, 2008.

Bohé, A.: The existence of supersensitive boundary-value problems, Methods and Applications of Analysis, 3(3), 318-334, 1996.

Denis, B., Laprise, R., and Caya, D.: Sensitivity of a regional climate model to the resolution of the lateral boundary conditions, Clim. Dynam., 20, 107-126, 2003.

Diaconescu, E. P., Laprise, R., and Sushama, L.: The impact of lateral boundary data errors on the simulated climate of a nested regional climate model, Clim. Dynam., 28, 333-350, 2007.

Errico, R. M.: What is an adjoint model?, B. Am. Metor. Soc., 78, 2577-2591, 1997.

Ferreira-Mendonca, L., Lopes, V. L. R., and Marinez, J. M.: QuasiNewton Acceleration for Equality Constrained Minimization, Comput. Optim. Appl., 40, 373-388, 2008.

Fisher, M., Nocedal, J., Tremolet, Y., and Wright, S. J.: Data Assimilation in Weather Forecasting: A Case Study in PDE-
Constrained Optimization, Technical Report, Optimization Technology Center, 2006.

Furihata, D.: Finite difference schemes for $\frac{\partial u}{\partial t}=\left(\frac{\partial}{\partial x}\right)^{\alpha} \frac{\delta G}{\delta u}$ that inherit energy conservation or dissipation property, J. Comput. Phys., 156, 181-205, 1999.

Gershunov, A., Barnett, T. P., Cayan, D. R., Tubbs, T., and Goddard, L.: Predicting and Downscaling ENSO Impacts on Intraseasonal Precipitation Statistics in California: The 1997/1998 Event, J. Hydrometeorol., 1, 201-210, 2000.

Griffin, D. A. and Thomson, K. R.: The adjoint method for data assimilation used operationally for shelf circulation, J. Geophys. Res., 101(C2), 3457-3477, 1996.

Griewank, A.: On Automatic Differentiation, Mathematical Programming: Recent Developments and Applications, Kluwer Academic Publishers, 83-108, 1989.

Grimshaw, R.: Korteweg-de Vries equation. Encyclopedia of Nonlinear Science, edited by: Scott, A. C., Taylor and Francis, New York, 504-511, 2004.

Kanamaru, H. and Kanamitsu, M.: Scale selective bias correction in a downscaling of global analysis using a regional model, California energy commission, PIER energy-related environmental research, CEC-500-2005-130, 2005

Korteweg, D. J and de Vries, H.: On the Change of Form of Long Waves advancing in a Rectangular Canal and on a New Type of Long Stationary Waves, Philosophical Magazine, 5th series, 36, 422-443, 1895.

Le Dimet, F.-X. and Talagrand, O.: Variational algorithms for analysis and assimilation of meteorological observations: theoretical aspects, Tellus, 38A, 97-110, 1986.

Leredde, Y., Lellouche, J.-M., Devenon, J.-L., and Dekeyser, I.: On initial, boundary and viscosity coefficient control for Burgers' equation, Int. J. Numer. Meth. Fluids, 28, 113-128, 1998.

Lorenc, A. C.: Modelling of error covariances by 4D-Var data assimilation, Q. J. Roy. Meteor. Soc., 129, 3167-3182, 2003.

Lorenc, A. C. and Payne, T.: 4D-Var and the butterfly effect: Statistical four-dimensional data assimilation for a wide range of scales, Q. J. Roy. Meteor. Soc., 133, 607-614, 2007.

Lu, C. and Browning, G. L.: Four-dimensional variational data assimilation for limited-area models: lateral boundary conditions , solution uniqueness, and numerical convergence, J. Atmos. Sci., 57, 1341-1353, 2000.

Martínez, J. M.: Practical quasi-Newton methods for solving nonlinear systems, J. Comput. Appl. Math., 124, 97-122, 2000.

Nocedal, J. and Wright, S. J.: Numerical Optimization, Springer Verlag, 1999.

Obukhov(Oboukhov), A. M.: On the question of the geostrophic wind, Izv. Akad. Nauk SSSR Ser. Geograf-Geofiz., 13, 281-306, 1949.

Rabier, F.: Overview of global data assimilation developments in Numerical Weather Prediction centres, Q. J. Roy. Meteor. Soc., 131, 3215-3233, 2005.

Rossby, C.-G.: Relation between variations in the intensity of the zonal circulation of the atmosphere and the displacements of the semi-permanent centers of action, J. Marine Res., 2, 38-55, 1939.

Seth, A., Rausher, S. A., Camargo, S. J., Quian, Jian-Hua, and Pal, J. S.: RegCM3 regional climatologies for South America using reanalysis and ECHAM global model driving fields, Clim. Dynam., 28, 461-480, 2007. 
von Storch, H., Langenberg, H., and Feser, F.: A spectral nudging technique for dynamical downscaling purposes, Mon. Weather Rev., 128, 3664-3673, 2000.

Waldron, K. M., Peagle, J., and Horel, J. D.: Sensitivity of a spectrally filtered and nudged limited-area model to outer model options, Mon. Weather Rev., 124, 529-547, 1996.
Zou, X. and Kuo, Y.-H.: Rainfall assimilation through an optimal control of initial and boundary conditions in limited-area mesoscale model, Mon. Weather Rev., 124, 2859-2882, 1996. 